\title{
Microradiosurgical cortical transections generated by synchrotron radiation
}

\author{
Pantaleo Romanelli a, b, Erminia Fardone c, 1 , Domenico Bucci ${ }^{\mathrm{d}}$, Giuseppe Battaglia ${ }^{\mathrm{d}}$, \\ Elke Bräuer-Krisch ${ }^{\mathrm{C}}$, Herwig Requardt ${ }^{\mathrm{C}}$, Geraldine Le Duc ${ }^{\mathrm{c}}$, Alberto Bravin ${ }^{\mathrm{c}, *}$ \\ a Cyberknife Radiosurgery Center, Centro Diagnostico Italiano, via Saint Bon, 20147 Milano, Italy \\ ${ }^{\mathrm{b}}$ AB Medica, 31 via Nerviano, 20020 Lainate, Milano, Italy \\ ${ }^{\mathrm{c}}$ European Synchrotron Radiation Facility (ESRF), 71 avenue des Martyrs, 38043 Grenoble, France \\ ${ }^{\mathrm{d}}$ I.R.C.C.S. Istituto Neurologico Mediterraneo Neuromed, Località Camerelle, 86077 Pozzilli, Italy
}

\section{A R T I C L E I N F O}

\section{Article history:}

Received 23 December 2014

Received in revised form

15 April 2015

Accepted 13 May 2015

Available online 29 May 2015

\section{Keywords:}

Brain cortex

Epilepsy

Microbeam

Synchrotron transections

\begin{abstract}
A B S T R A C T
Purpose: Microplanar X-ray beams (microbeams) originated by synchrotron sources have been delivered to the visual brain cortex regions in rodents to create microscopically narrow lesions. The effects of microbeams mimic those generated by microsurgical subpial transections (also known as multiple subpial transections) but are obtained in a low-invasive way.

Methods: Image-guided atlas-based microbeam cortical transections have been generated on seven 1 month-old Wistar rats. An array of 10 parallel beams of 25 microns in thickness and spaced of 200 micron center-to-center was centered on the visual cortex and deposited an incident dose of $600 \mathrm{~Gy}$. Results: The procedure was well tolerated by rats. After recovery, rats showed regular behavior, no sign of gross visual impairment and regular weight gain. After 3 months, rats were sacrificed and brains histologically examined. Cortical transections resembling those obtained through a surgical incision were found over the irradiated region. Remarkable sparing of the cortical columns adjacent to the transections was observed. No sign of radionecrosis was evident at least at this time point.

Conclusions: The visual brain cortex transected by synchrotron-generated microbeams showed an incision-like path of neuronal loss while adjacent non irradiated columns remained intact. These preliminary findings, to be further investigated also using other techniques, suggest that microbeam radiosurgery can affect the cortex at a cellular level providing a potential novel and attractive tool to study cortical function.

(C) 2015 Published by Elsevier Ltd on behalf of Associazione Italiana di Fisica Medica. This is an open access article under the CC BY-NC-ND license (http://creativecommons.org/licenses/by-nc-nd/4.0/).
\end{abstract}

\section{Introduction}

Synchrotron-generated X-ray microplanar beams (microbeams) are emerging as a novel tool to generate focal brain lesions in a lowinvasive way [1].

The minimal beam divergence of synchrotron $\mathrm{x}$-rays allows the delivery of extremely high doses of radiation to the cells along the penetration path with minimal dose dispersion over the adjacent

\footnotetext{
* Corresponding author. Tel.: +33 476882843.

E-mail addresses: radiosurgery2000@yahoo.com (P. Romanelli), efardone@ neuro.fsu.edu (E. Fardone), domenico.bucci@neuromed.it (D. Bucci), giuseppe. battaglia@neuromed.it (G. Battaglia), brauer@esrf.fr (E. Bräuer-Krisch), requardt@ esrf.fr (H. Requardt), bravin@esrf.fr (A. Bravin).

1 Present address: Florida State University, Department of Biological Science, Tallahassee, Florida, USA.
}

cells. Fall-out is extremely brisk: the doses received by the cells adjacent to a microbeam path are reduced by two orders of magnitudes within few dozen or hundreds of microns depending on the microbeam size [2]. This extremely selective spatial delivery allows to irradiate microscopic slices of tissue with doses in the range of several hundreds of Grays, generating a quasi-surgical cuts sized from 25 to 600 microns. We have observed that microbeam arrays delivered to the cortex generate cortical transections show histological effects similar to transections used in epilepsy surgery (multiple subpial transections, MST) [3,4], although transections are not limited to the visual cortex but extends to the hippocampus and basal cortex.

The placement of a surgical incision tangential to the cortex aims at disconnecting adjacent cortical columns by cutting the horizontal axons subserving seizure spreading while preserving the vertical axons directed to the basal ganglia and the spinal cord. 
We explored here the histological effects of microbeam cortical transections generated by X-ray beams of microscopic thickness and spacing and performed over the visual cortex. This brain region cannot be approached by conventional surgical means, thus banning the treatment of medically-refractory seizure foci here located. More in general, the ability to generate focal lesions or microscopic transections over eloquent and non-eloquent cortex in experimental animals is of great interest for the development of new experimental models in neurobiology; additionally, this technique also opens new treatment avenues for a variety of neuropsychiatric disorders originating from focal brain dysfunction.

\section{Methods}

\section{Animals}

Male Wistar rats (250-275 g) were purchased from Charles River Laboratories (L'Asbresle, France). Rats were kept under environmentally controlled conditions (ambient temperature $=22{ }^{\circ} \mathrm{C}$, humidity $=40 \%$ ) on a 12 -h light/dark cycle with food and water ad libitum.

All operative procedures related to animal care strictly conformed to the guidelines of the French Government under licenses 380324 and $\mathrm{A} 3818510002$.

\section{Irradiation source}

Synchrotron-generated X-ray beams are tangentially emitted by relativistic electron bunches circulating in the storage ring of a synchrotron radiation facility. The experiments were carried out at the ID17 beamline of the European Synchrotron Radiation Facility (ESRF, Grenoble, France). The X-ray source is a wiggler (a magnetic structure of alternating poles positioned on a straight section of the storage ring, consisting of a sequence of 21 poles, with a period of $150 \mathrm{~mm}$ ) producing a wide spectrum of photons with an energy range up to several hundreds of kilo-electronvolts (keV). The X-ray beam is quasi-parallel (horizontal divergence $\leq 1 \mathrm{mrad}$, vertical divergence $\leq 0.02 \mathrm{mrad}$ ) and possesses a quasi laminar shape (maximum beam size at the sample position: $40 \mathrm{~mm} \times 1 \mathrm{~mm}$, horizontal $\mathrm{x}$ vertical).

After filtering with a sequence of beryllium $(0.5 \mathrm{~mm})$, carbon $(1.5 \mathrm{~mm})$, aluminum $(1.5 \mathrm{~mm})$ and copper $(1.0 \mathrm{~mm})$, the polychromatic radiation spectrum entering the treatment room has a mean energy of approximately $100 \mathrm{keV}$ when a magnetic field of 1.6T is applied to the wiggler, as in this case [2]. The beam is spatially fractionated into an array of rectangular microbeams of variable size (25-100 microns) by means of a multislit collimator [5]. The extremely X-ray fluence produced by the wiggler determines an entrance dose rate of around $16,000 \mathrm{~Gy} / \mathrm{s}$ [6], allowing the deposition in a fraction of second of doses of hundreds of Grays along the microscopic planes.

\section{Irradiation geometry}

An array of 10 parallel microbeams depositing an incident dose of $600 \mathrm{~Gy}$ was placed over the visual cortex (V2ML, V2L) [7]. The nominal microbeam size as created by the collimator was $25 \pm 3 \mu \mathrm{m}$ and the center-to-center spacing was $200 \pm 1 \mu \mathrm{m}$ at the collimator position. Due to the beam divergence, at the sample position the beam size was increased to an average value of $26.3 \mu \mathrm{m}$ and the center-to-center to $211 \mu \mathrm{m}$, providing a nominal mediolateral coverage of the irradiated cortex of $1.924 \pm 0.002 \mathrm{~mm}$. The first beam was placed on the right hemisphere, centered $3.0 \pm 0.2 \mathrm{~mm}$ lateral to the midline. The microbeam length in the anteroposterior (AP) direction was equal to $1 \mathrm{~mm}$, and was positioned starting at
$4.8 \mathrm{~mm}$ and ending $5.8 \mathrm{~mm}$ behind the bregma. Figure 1 illustrates a simulation of the beam array placement.

\section{Image-guided beam delivery}

Rats were placed vertically and fixed by ear bars and teeth on a home-made plexiglas stereotactic frame and placed on a Kappatype goniometer (Huber, Germany), by which the rat could be translated and rotated in front to the fixed horizontal X-ray beam [6].

To guide irradiations, low dose high resolution radiographs were acquired by vertically scanning the sample in front of the attenuated polychromatic X-ray beam (wiggler magnetic field set at $\mathrm{B}=0.32 \mathrm{~T}$ ) and by recording images using a $2048 \times 2048$ pixel Frelon camera [8], placed about $5 \mathrm{~m}$ downstream the sample. Radiographs allowed identifying the bregma and therefore to center microbeams on the desired target (see Fig. 2).

For irradiations, the beam height was defined by a $520 \mu \mathrm{m}$ wide tungsten slit, placed at $1 \mathrm{~m}$ upstream the animal. The dose was delivered by vertically moving the Kappa goniometer, at a speed inversely proportional to the dose to be delivered. Each irradiation lasted less than $1 \mathrm{~s}$. The vertical irradiation field was determined by the opening-closing of fast shutters, located $7 \mathrm{~m}$ upstream the rats and synchronized with the movement of the kappa goniometer. All movements were remotely controlled and irradiation values were preset by the operator before the treatment. The animal immobility during exposure was checked on 3 high resolution cameras located in the control hutch. The incoming spatially non-fractionated dose was measured using an ionization chamber and the mid-valley doses were calculated by Monte Carlo simulations.

\section{Dose profile}

The dose profile into the target consists of an alternation of peaks and valleys, i.e., of high doses along the microbeam path and low doses in the spaces between them. The reference valley dose is normally assumed as the minimum dose in between two microbeams. The valley dose must not exceed the maximum dose tolerated by the normal tissues traversed by the radiation; its value depends on several parameters including the photon energy, number of microbeams, microbeam width and height, the centerto-center distance, as well as the depth, shape, and composition of tissues proximal to, within, and distal to the target. The dose deposited by the microbeams (600 Gy) refers to the peak entrance dose measured at $3 \mathrm{~mm}$ in depth; it is constantly decreasing in the target with a half value layer of $\sim 4.5 \mathrm{~cm}$ in water. The Monte Carlo calculated valley doses [9] are as low as $4.4 \mathrm{~Gy}$ at $3 \mathrm{~mm}$ depth with a kind of build up reaching $5.3 \mathrm{~Gy}$ at $1 \mathrm{~cm}$ in depth. Figure 3 shows on a logarithmic scale the two different profiles for the peak and the valley dose in the target.

\section{Behavioral observation}

Behavioral observation was performed with the rats inside their transparent plexiglas cage. Motor activity, exploratory activity, fur conditions and access to food and water were monitored for a period of 10 min every 7 days for three months following irradiation. Weight gain was also assessed weekly.

\section{Histology}

Rats were randomly chosen and killed by a Dolethal ${ }^{\circledR}$ overdose. Fifteen micron thin coronal brain sections were cut at $-22^{\circ} \mathrm{C}$ on a cryostat (Microm HM560, France); in order to mark neurons and glia, slices were stained for 8 min with thionin (Nissl staining) and 


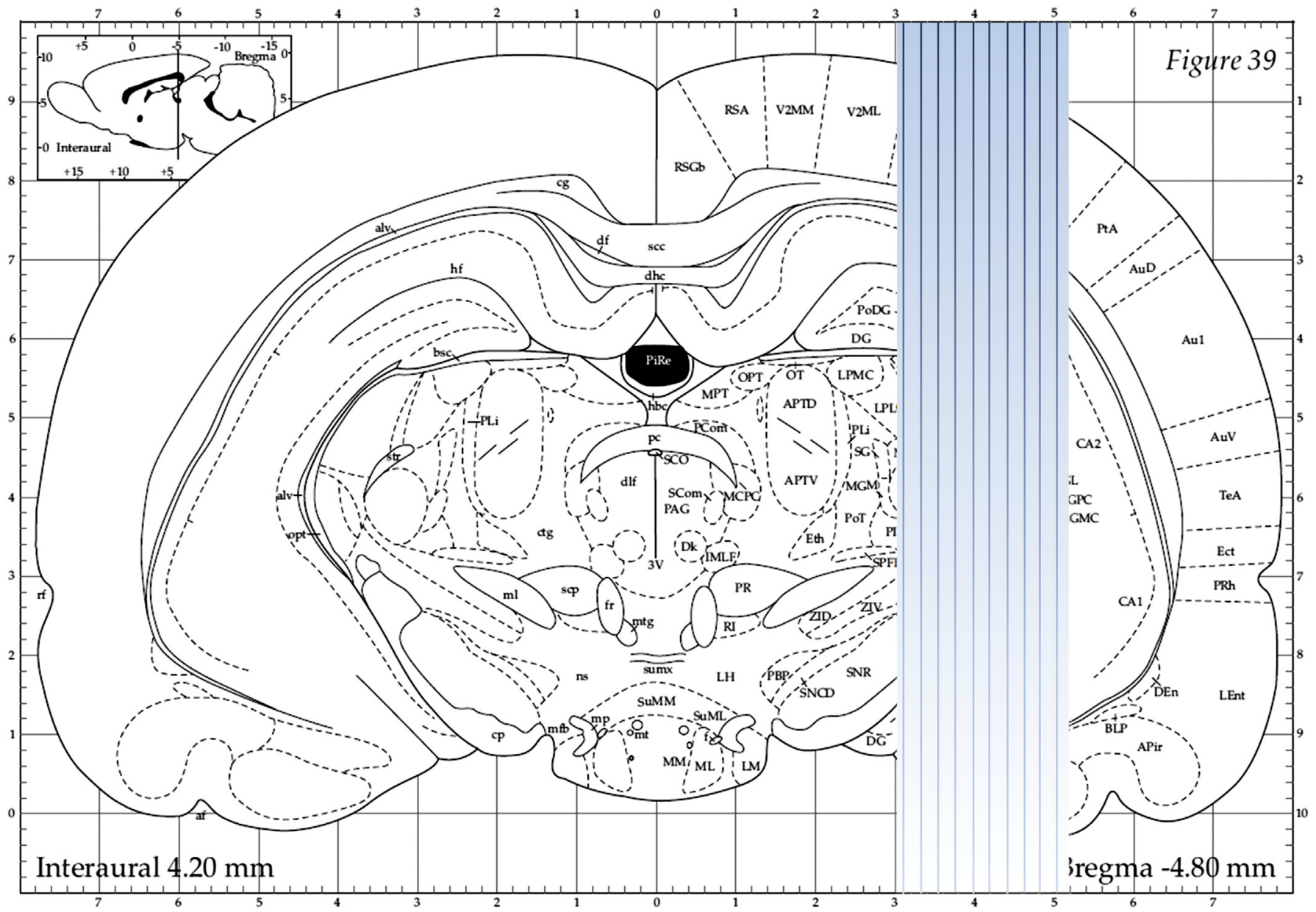

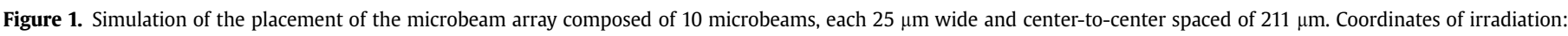
Antero posterior: from -4.8 to -5.8 from the bregma; lateral (right hemisphere): from 3.0 to $4.9 \mathrm{~mm}$.

then dehydrated in alcohol series, cleared in toluene, and coverslipped with Pertex (Medite, Burgdorf, Germany).

\section{Results}

\section{Weight and behavioral follow-up}

Animals showed regular behavior with no signs of irritability, apathy, fur alterations or gross visual impairment. Exploratory activity was regular as compared with control animals. Weekly assessment of body weight did not show any difference vs. control animals (not shown).

\section{Histology}

Nissl staining performed 3 months after the irradiation was remarkable for the absence of visible radionecrosis at this time point, although it can develop to later times up to 1 year, over the irradiated visual cortex, despite the extremely high radiation doses delivered. We observed a normal architecture of irradiated brain regions, with a regular and well preserved distribution of cells within the primary visual cortex and all other regions between the microbeam paths. Cells were absent just along the irradiation path in the visual cortex (Fig. 4) and all other brain regions traversed by the microbeams (Fig. 1). This pattern of stripes (absence of cells along the microbeam path alternating with well preserved neurons outside and in between the paths) was consistently observed
(Fig. 4). The lack of cells was not limited to the visual cortex but extended to the entire brain regions crossed by microbeams such as the hippocampal region and the basal the basal cortex (see Fig. 1). In essence, the treatment generated microscopic incisions along the visual cortex sparing entirely the cortical columns adjacent to the microbeam paths.

\section{Discussion}

The sparing effect of synchrotron-generated microbeams has been extensively studied in the past years on different types of animal models (mice, rats, piglets) (see for instance [10-12]) on chorioallontoic membrane [13] and in-ovo [14] both at the ESRF (Grenoble, France) and at the BNL (Brookhaven, Upton, NY) aiming at verifying the safe use of high doses of microbeams to potentially treat aggressive brain tumors. More recently, microbeams carrying doses of several hundreds of Grays provided a novel minimally invasive tool to transect the cortex: the equivalent of a surgical incision is generated across the cortex while the adjacent tissue retains its normal histological architecture [1].

Microbeam cortical transections are a highly innovative method to study basic cortical functions but can also be used to treat epileptogenic cortex. The smallest beam size produced by currently available stereotactic radiosurgery devices is about $5 \mathrm{~mm}$ [15]. A modern synchrotron source can instead deliver highly intense X-ray microbeam arrays of microscopic thickness down to $25 \mu \mathrm{m}$. 


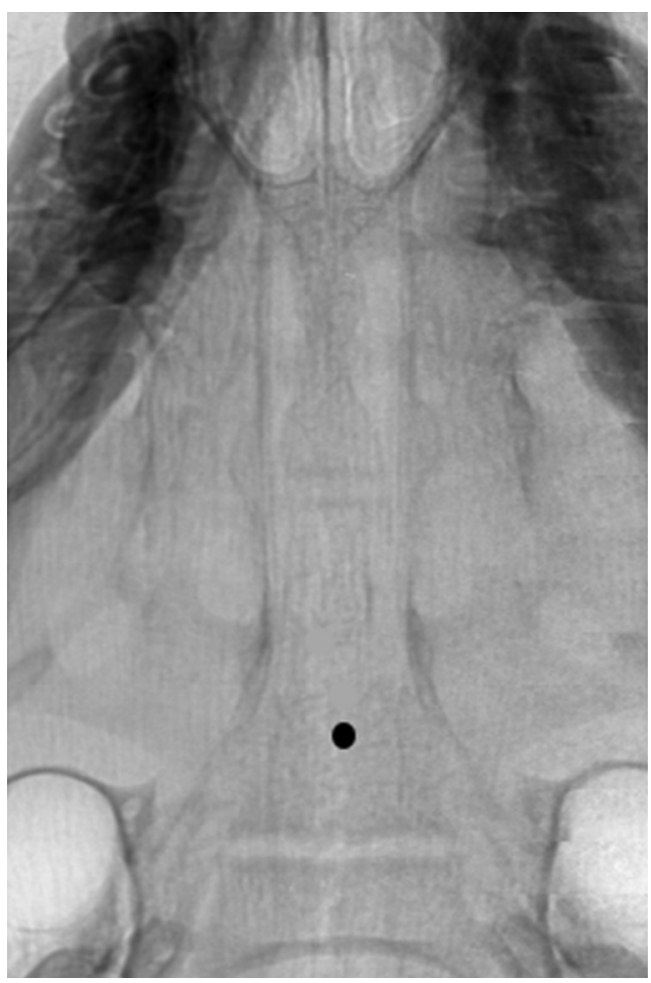

Figure 2. X-ray radiograph of the rat before microbeam irradiation to identify the position of the bregma (dark circle).

The subsecond delivery of high dose of synchrotron radiation Xrays (dose rates about six times larger than those available with conventional sources) to microscopic slices of tissue generates cell death only along the path of penetration and in the near vicinities, leaving intact the adjacent cells [14], [16-18]. Doses up to $4000 \mathrm{~Gy}$ can be delivered through microscopic beams sized $25-60 \mu \mathrm{m}$ with no histological evidence of widespread radionecrosis outside the penetration path. This exceptional resistance of the normal tissue to high doses is usually indicated with "tissue sparing effect".

The immunohistochemical studies reported previously $[1,10$, 19], show that the neurons hit by the microbeam along its penetration path die almost immediately while the adjacent cells outside the high dose volume remain viable. Progressively lower doses (but still much higher than conventional radiosurgical or radiotherapeutic doses) are required to avoid tissue damage if ticker beams (100-600 $\mu \mathrm{m})$ are used. Submillimetric beams (sized

\section{peak and valley dose profiles in depth}

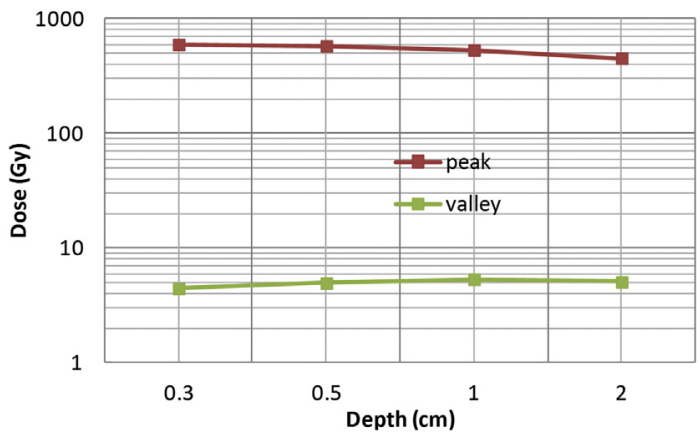

Figure 3. The Monte Carlo calculated peak and valley dose profiles at different depths in the target.

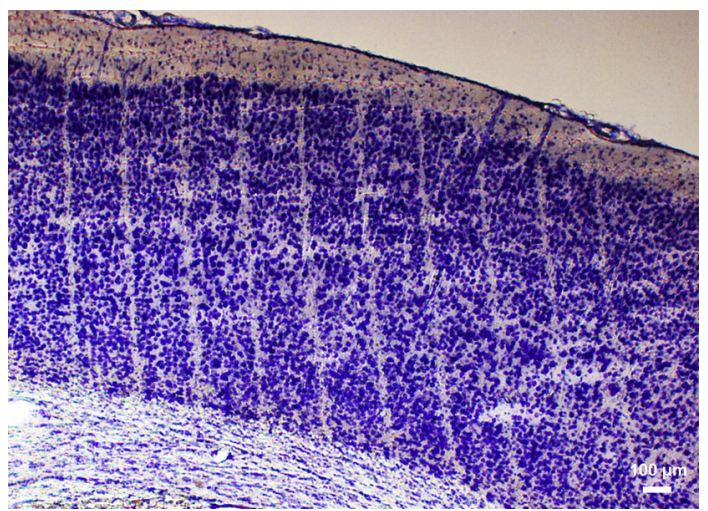

Figure 4. Nissl staining of the visual cortex of a representative rat irradiated with microbeams. Note the typical pattern of alternating stripes (absence of cells along the microbeam path alternating with well preserved cell distribution outside and between the paths).

0.6-0.7 $\mathrm{mm}$ ) appear to retain the tissue sparing effect allowing the delivery of incident doses of $400 \mathrm{~Gy}$ to the spinal cord of rats without inducing neurological damage [11]. Irradiation of rat spinal cord with four parallel $0.68 \mathrm{~mm}$ thick microbeams at $400 \mathrm{~Gy}$ indepth beam dose did not induce paralysis after 7 months in three out of four rats [20]. This study showed not only that a highly radiosensitive structure such as the spinal cord can receive high dose irradiation through a microbeam array without neurologic sequelae but also that a beam width up to $0.68 \mathrm{~mm}$ is well tolerated, substantially maintaining the tissue sparing properties of thinner beams.

The ability of microbeam arrays to avoid radionecrosis and to preserve the architecture of the irradiated tissue is mainly attributed to the rapid regeneration of normal microvessels. Only a short segment of the microvascular bed receives ablating doses while the adjacent endothelial cells fall into the valley dose region receiving just a few Grays and can restore quickly the continuity of vascular supply [13]. The wide spatial interface between the unhindered tissue placed in the valleys and the tissue irradiated with peak doses within the microbeam paths facilitates a widespread vascular recolonization of the tissue receiving necrotic doses preventing the dissolution of the architecture of the irradiated tissues [21]. The self-repair of the normal microvasculature through the migration of unaffected cells surrounding the paths of microbeam penetration is considered by most as the basis for this ability of normal tissue to tolerate high dose microbeam irradiation [19].

After having studied the effects of microbeam transections over sensorimotor cortex [1,22], we analyze here the histological effects of microbeam transections performed over the visual cortex in a group of 7 healthy rats. This experiment confirmed the remarkable ability of synchrotron-generated microbeams to ablate slices of tissue while sparing the adjacent cells. The ability to generate in a non- or low-invasive way the equivalent of microsurgical cortical transections provides a new and attractive tool to modulate cortical function in healthy and epileptic brain. The horizontal fibers connecting nearby and distant cortical columns are an essential pathway for the spreading of seizures [1]. The severing of these connections leads to a parcellization and disconnection of the epileptic focus with consequent seizure control while the functions carried by the vertical axons are spared [1]. Aside from the tight dosimetry, the low energy of monochromatic beams makes them well suited to treat superficial targets such us the cortex. Microbeam transections, either placed over neocortical seizure foci or through the hippocampus, could prove to be an excellent tool to be added to the current radiosurgical techniques used to control 
seizures. A series of experiments are currently planned at ESRF aiming at the development of an experimental model of microbeam cortical transections in epileptic rats and verify the ability of microbeam cortical transection to control seizures without damage to the eloquent cortex irradiated.

\section{Conclusions}

The irradiation of normal brain with microbeam arrays is characterized by a distinct tissue-sparing effect. Peak doses between 300 and $600 \mathrm{~Gy}$ delivered through microscopic beams are well tolerated by the CNS with no histological evidence of widespread brain damage. These preliminary findings obtained in the microbeam irradiated visual cortex of rats, associated to previous published results on the tissue sparing effect of microbeams may suggest the use of microbeam arrays to induce transections along cortex and other brain regions aiming at modulating cortical function through the selective cutting of horizontal axons connecting adjacent columns. Both sensorimotor cortex and visual cortex tolerate well the placement of synchrotron-generated transections. These microradiosurgical transections offer an attractive low-invasive tool to study the basic functions of the cortex and to treat epileptic foci located in eloquent and non-eloquent cortex.

\section{Acknowledgments}

Authors acknowledge the ESRF for provision of beamtime at ID17 and T. Brochard, D. Dallery, M. Renier, C. Nemoz for their kind support during experiments. This work has been performed with the support of the COST Action TD1205 (SYRA3) which is warmly acknowledged.

\section{References}

[1] Romanelli P, Fardone E, Battaglia G, Brauer-Krish E, Prezado Y, Requardt E, et al. Synchrotron-generated mirobeam sensorimotor cortex transections induce seizure control without disruption of neurological function. PlosOne 2013;8(1):e53549.

[2] Siegbahn EA, Bräuer-Krisch E, Stepanek J, Blattmann H, Laissue JA, Bravin A Determination of dosimetrical quantities used in microbeam radiation therapy (MRT) with Monte Carlo simulations. Med Phys 2006;33:3248-59.

[3] Morrell F, Whisler WW, Bleck TP. Multiple subpial transection: a new approach to the surgical treatment of focal epilepsy. J Neurosurg 1989;70: 231-9.

[4] Orbach D, Romanelli P, Devinsky O, Doyle W. Late seizure recurrence after multiple subpial transections. Epilepsia 2001;42:1130-3.
[5] Bräuer-Krisch E, Requardt H, Brochard T, Berruyer G, Renier M, Laissue JA, et al. New technology enables high precision multislit collimators for microbeam radiation therapy. Rev Sci Instrum 2009;80(7):074301.

[6] Bräuer-Krisch E, Serduc R, Siegbahn EA, Le Duc G, Prezado Y, Bravin A, et al. Effects of pulsed, spatially fractionated, microscopic synchrotron X-ray beams on normal and tumoral brain tissue. Mutat Res 2010;704:160-6.

[7] Paxinos G, Watson C. The rat brain in stereotaxic coordinates. 2nd ed. , London: Academic Press; 1986.

[8] Coan P, Peterzol A, Fiedler S, Ponchut C, Labiche JC, Bravin A. Evaluation of imaging performance of a taper optics CCD FReLoN' camera designed for medical imaging. J Synchrotron Radiat 2006;13:260-70.

[9] Fardone E. A new application of microbeam radiation therapy (MRT) on the treatment of epilepsy and brain disorders. In: PhD thesis. University of Grenoble; 2013.

[10] Serduc R, Vérant P, Vial JC, Farion R, Rocas L, Rémy C, et al. In vivo two-photon microscopy study of short-term effects of microbeam irradiation on normal mouse brain microvasculature. Int J Radiat Oncol Biol Phys 2006;64(5): $1519-27$.

[11] Dilmanian FA, Zhong Z, Bacarian T, Benveniste H, Romanelli P, Wang R, et al Interlaced $\mathrm{x}$-ray microplanar beams: a radiosurgery approach with clinical potential. Proc Natl Acad Sci U S A 2006;103:9709-14.

[12] Laissue JA, Blattmann H, Wagner HP, Grotzer MA, Slatkin DN. Prospects for microbeam radiation therapy of brain tumours in children to reduce neurological sequelae. Dev Med Child Neurol 2007;49:577-81.

[13] Sabatasso S, Laissue JA, Hlushchuk R, Graber W, Bravin A, Bräuer-Krisch E et al. Microbeam radiation induced tissue damage depends on the stage of vascular maturation. Int J Radiat Onc Biol Phys 2011;80(5):1522-32.

[14] Dilmanian FA, Morris GM, Le Duc G, Huang X, Ren B, Bacarian T, et al. Response of avian embryonic brain to spatially segmented X-ray microbeams. Cell Mol Biol (Noisy-le-grand) 2001;47:485-93.

[15] Francescon P, Kilby W, Satariano N, Cora S. Monte Carlo simulated correct factors mach specific reference field dose calibration output factor measurement using fixed and iris collimators on the CyberKnife System. Phys Med Bio 2012;57(12):3741-58.

[16] Dilmanian FA, Morris GM, Zhong N, Bacarian T, Hainfeld JF, Kalef-Ezra J, et al. Murine EMT-6 carcinoma: high therapeutic efficacy of microbeam radiation therapy. Radiat Res 2003;159:632-41.

[17] Miura M, Blattmann H, Bräuer-Krisch E, Bravin A, Hanson AL, Nawrocky MM, et al. Radiosurgical palliation of aggressive murine SCCVII squamous cell carcinomas using synchrotron-generated X-ray microbeams. Br J Radiol 2006;79:71-5.

[18] Zhong Z, Morris GM, Bacarian T, Rosen EM, Dilmanian FA. Response of rat skin to high-dose unidirectional X-ray microbeams: a histological study. Radiat Res 2003; $160: 133-42$.

[19] Serduc R, Christen T, Laissue J, Farion R, Bouchet A, Bv Sanden, et al. Brain tumor vessel response to synchrotron microbeam radiation therapy: a shortterm in vivo study. Phys Med Biol 2008;53:3609-22.

[20] Dilmanian FA, Ou Y, Feinendegen LE, Peña LA, Bacarian T, Henn FA, et at. Tissue-sparing Eff X-ray microplanar beams Part CNS: is a bystander Eff involved? Exp Hematol 2007;35(4 Suppl 1):69-77.

[21] Prezado Y, Thengumpallil S, Renier M, Bravin A. X-ray energy optimization in minibeam radiation therapy. Med Phys 2009;36:4897-902.

[22] Anschel D, Romanelli P, Bravin A, Qhanum F, Bräuer-Krisch E, Le Duc G. VideoEEG analysis following microradiosurgical transections of rat sensorimotor cortex seizure focus. Cureus 2013;5(11):e145. 